\title{
Preparation and Execution of the Electrical Quality Assurance Program On the LHC Superconducting Circuits During the Second Long Shutdown
}

\author{
Jaromir Ludwin ${ }^{\circledR}$, Mateusz Bednarek ${ }^{\circledR}$, Mikolaj Bednarski ${ }^{\circledR}$, Jaroslaw Bielewski ${ }^{\circledR}$, Pawel Bogdali, \\ Giorgio D’Angelo, Witold Daniluk, Erazm Maria Dutkiewicz, Dominik Grzadziel, Grzegorz Janik, Piotr Jurkiewicz, \\ Wieslaw Kantor, Andrzej Kotarba ${ }^{\circledR}$, Artur Krempa ${ }^{\circledR}$, Pawel Lasko ${ }^{\circledR}$, Waclaw Ostrowicz ${ }^{(}$, \\ Andrzej Proszkowiec ${ }^{\circledR}$, Felix Rodriguez Mateos, Andrzej Siemko, Magda Szeliga, Karol Witkowski, \\ and Damian Wojas
}

\begin{abstract}
The electrical quality assurance (ELQA) of the superconducting circuits of CERN's Large Hadron Collider (LHC) during its second Long Shutdown (LS2) requires the execution of vast measurement campaigns. Each of 1658 electrical circuits as well as the instrumentation of each of the $\mathbf{1 7 4 6}$ individual cryo-assemblies housing magnets, bus-bars and other components of the superconducting circuits, has to undergo a series of tests. At the beginning of the shutdown the tests are performed before and after the warm-up from cryogenic temperatures. The same tests are repeated at the end of the shutdown, this time before and after the cool-down. As part of the dipole bypass diode insulation consolidation project and due to the replacement of a few non-conform magnets, additional electrical tests and measurements had to be performed throughout the shutdown period. With each measurement, electrical parameters like resistance, complex impedance, and the quality of the insulation are verified and stored for future reference. Due to the many thousands of measurements to be performed, multiple automated mobile test benches had to be developed for the different test types along with dedicated database and analysis software applications for the documentation and follow-up of the test results and the non-conformities observed.
\end{abstract}

Index Terms-Circuit testing, Large Hadron Collider (LHC), quality assurance, superconducting magnets.

\section{INTRODUCTION}

$\mathbf{T}$ HE COLLABORATION between CERN and the Henryk Niewodniczanski Institute of Nuclear Physics Polish

Manuscript received November 29, 2020; revised February 11, 2021; accepted March 3, 2021. Date of publication March 17, 2021; date of current version May 24, 2021. (Corresponding author: Jaromir Ludwin.)

Jaromir Ludwin is with the Henryk Niewodniczanski Institute of Nuclear Physics Polish Academy of Sciences, Krakow, Poland, and also with CERN, Geneva, Switzerland.

Mateusz Bednarek, Giorgio D’Angelo, Felix Rodriguez Mateos, and Andrzej Siemko are with CERN, Geneva, Switzerland (e-mail: mateusz. bednarek@cern.ch; giorgio.dangelo@cern.ch; felix.rodriguezmateos@ cern.ch; andrzej.siemko@cern.ch).

Mikolaj Bednarski, Jaroslaw Bielewski, Pawel Bogdali, Witold Daniluk, Erazm Maria Dutkiewicz, Dominik Grzadziel, Grzegorz Janik, Piotr Jurkiewicz, Wieslaw Kantor, Andrzej Kotarba, Artur Krempa, Pawel Lasko, Waclaw Ostrowicz, Andrzej Proszkowiec, Magda Szeliga, Karol Witkowski, and Damian Wojas are with the Henryk Niewodniczanski Institute of Nuclear Physics Polish Academy of Sciences, Krakow, Poland and also with CERN, Geneva, Switzerland.

Color versions of one or more figures in this article are available at https: //doi.org/10.1109/TASC.2021.3065978.

Digital Object Identifier 10.1109/TASC.2021.3065978
Academy of Sciences (IFJ PAN) in Krakow, in the field of ELQA dates back to 2004 and the construction phase of the LHC [1]. The two organizations continued their collaboration during the 2009 LHC consolidation period and during the first planned Long Shutdown (LS1), which started in 2013. Following the successful LS1 test campaigns, at the end of 2014 CERN and IFJ PAN established a collaboration agreement, which provided a framework for further joint efforts in ELQA hardware and software upgrades, development of new methods, as well as next measurement and test campaigns during technical stops and LS2.

\section{TESTS REQuiRED FOR ELQA}

The ELQA tests performed on superconducting circuits can be divided into three categories:

- High voltage insulation qualifications - performed on complete superconducting circuits and independently on quench heaters with their cabling. Each test is conducted by applying a controlled voltage and measuring resulting leakage current. In the majority of cases, the test is performed using a dedicated multichannel device, described in [2].

- Continuity, voltage tap sequence checks, and resistance measurements - performed on complete superconducting circuits as well as on parts of circuits, e.g., individual magnets, or their instrumentation such as voltage taps and quench heaters. Each test can be composed of several to few hundreds automatically performed resistance measurements using equipment described in [3], [4], [5], [6], [7], and [9].

- Complex impedance measurements - performed on complete superconducting circuits or individual magnets, at frequencies from $1 \mathrm{~Hz}$ to $100 \mathrm{kHz}$ and stimulus current below $1 \mathrm{~A}$ peak. A dedicated gain/phase analyzers are integrated in the test systems and the same automated switching circuitry as for resistance measurements is utilized in this test. Details of the setup are described in [3] and [6].

All measurement data are checked against predefined thresholds and historical values [4] in order to obtain the test result. 
Both, raw data and test results are subsequently stored in the centrally hosted relational database, from where they can be accessed by follow-up and batch analysis software applications.

\section{PREPARATION FOR LS2 (2015 - 2018)}

In the years 2015 - 2018 three major ELQA-related upgrade projects required for LS2 were executed:

1) Upgrade of the multichannel high voltage insulation tester and production of eleven new units. The project included increasing test voltage, improving maintainability, and adding new safety features.

2) Upgrade of the automatic measurement system for validation of the interconnections between superconducting magnets [7]. This system is used whenever an LHC magnet is replaced and about 70 pairs of superconducting bus bars have to be reconnected. Within this project, we have produced hardware for four new measurement systems and developed dedicated software applications. The upgraded system is capable of performing automated, precise, four-wire measurements of resistance between extremities of superconducting bus bars and checking their distribution on the connection board. These measurements are performed before and after soldering of the main bus-bars and ultrasonic welding of the auxiliary bus-bars. The measurement points can be up to $500 \mathrm{~m}$ apart.

3) Following the hardware upgrade of the main mobile ELQA test bench in 2012 described in [6] and with experiences gained during LS1, a number of improvements was introduced in the low voltage measurement system. In the software part, the upgrades provided improved precision for voltage tap sequence identification, an additional four-wire resistance measurement performed on each coil of main circuits, and general code optimizations. Additionally, an external active filter has been developed, which further improved precision of measurements of low bus bar resistances at relatively low currents.

The activities carried out during technical stops of the LHC, which included replacement of one magnet, full recommissioning of an entire LHC sector, several small measurement campaigns, and special measurements related to existing non-conformity investigations were an important aspect of preparations for the LS2. While strictly required from the perspective of maintaining electrical quality of the superconducting circuits, those activities also provided a great opportunity to train new members of the ELQA team and to maintain the core of the team at full operational capacity.

\section{LS2 MEASUREMENT CAMPAIGNS (2018 - 2021)}

\section{A. Tests Execution}

For the purpose of the LS2 work management, a number of tasks was defined, including:

- Set of so-called "standard ELQA" measurements performed on each LHC superconducting circuit and on instrumentation of each superconducting magnet before and after warm-up of the machine at the beginning of LS2,

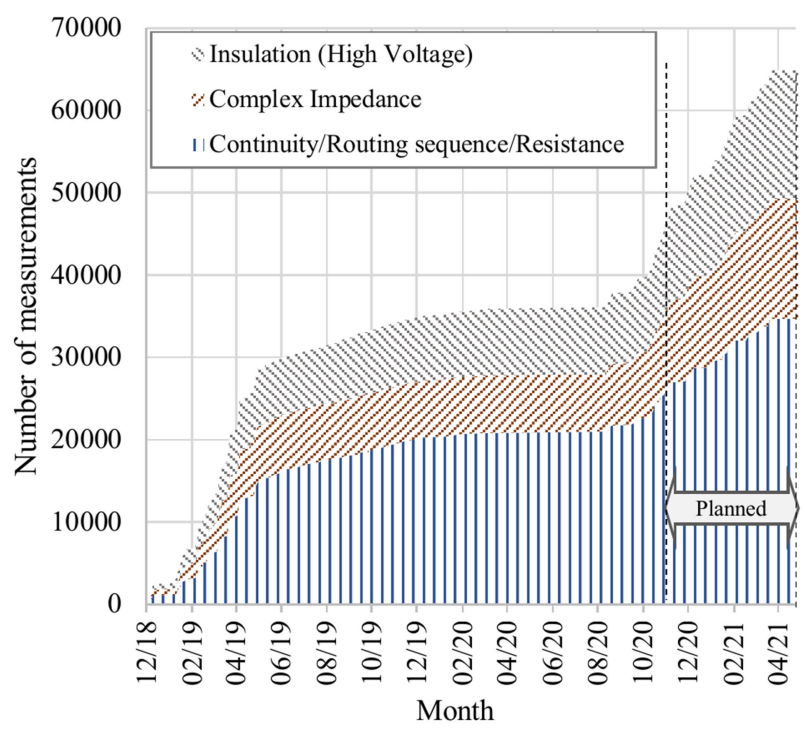

Fig. 1. Number of ELQA measurements during LS2.

- Development of high precision resistance measurement system for the main dipole protection by-pass diode lead contacts [9],

- Dedicated measurement campaigns during insulation consolidation of main dipole protection by-pass diodes monitoring of the contact resistances at each step of the consolidation and regular checks of the insulation,

- Tests related to replacement and reconnection of 22 superconducting magnet assemblies and 2 connection cryostats,

- Set of "standard ELQA" measurements performed on each LHC superconducting circuit and on instrumentation of each superconducting magnet before and after cool-down of the machine at the end of LS2.

In the peak of activities there were 25 collaborators from IFJ PAN in the ELQA team, including test engineers and technicians. As shown in Fig. 1 the number of the ELQA tests and measurements related to LS2 activities is of the order of 48400 on $29^{\text {th }}$ November 2020, and is expected to be more than 64500 at the end of LS2.

\section{B. Protection Diode Lead Contacts Resistance Measurement}

An example of large scale measurements performed by the ELQA team during LS2 is the set of measurements performed on protection by-pass diode lead contacts.

Each of eight LHC sectors contains one main dipole circuit, composed of 154 superconducting magnets connected in series, with a total inductance of $15 \mathrm{H}$ and nominal current of 11.8 $\mathrm{kA}$. Each of the 1232 magnets is equipped with a protection by-pass diode, connected in parallel to the magnet coil using dedicated copper bus bars. The diodes take over the current of the corresponding dipoles in case of magnet quench and are by-passing electrically the magnets until the energy stored in the magnetic field of the whole circuit is extracted. The diodes are operating in the cryogenic conditions as they share the volume with superconducting components of the magnet. 


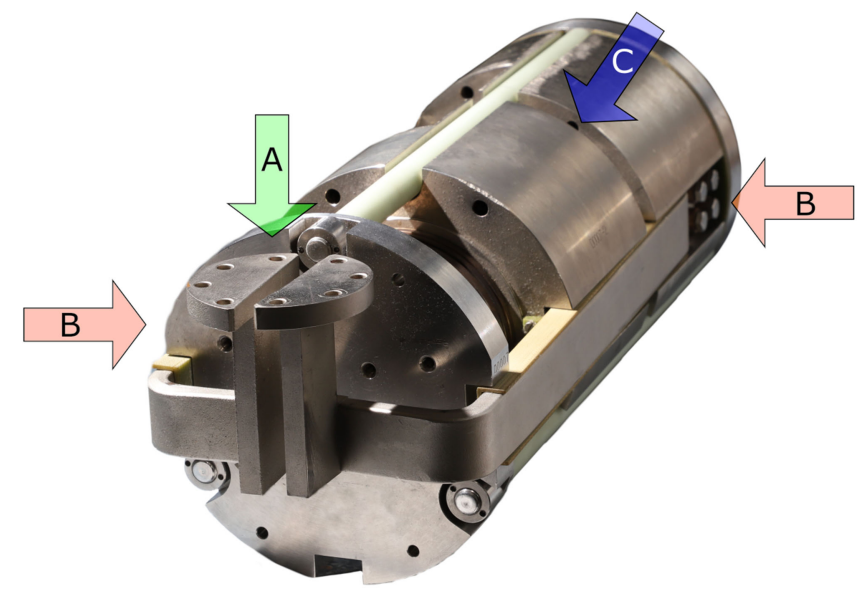

Fig. 2. Bypass protection diode of the LHC main dipole magnet. Bolted and compression contacts marked with arrows: A - two "half-moon" bolted contacts, $\mathrm{B}$ - two bolted bus bar to heat sink contacts, $\mathrm{C}$ - two compression based diode to heat sink contacts.

During LS2 the electrical insulation system of all protection diodes in the main dipole circuits had to be reinforced [8].

The connection between the superconducting circuit and the by-pass diode contains six contacts as shown in Fig. 2, which have to be able to carry the current of the circuit during a magnet quench and the subsequent circuit discharge. As the diode assembly was manipulated during the insulation reinforcement activity, a dedicated measurement system [9] had to be developed and measurements of all contacts at four different stages of the intervention had to be performed in order to assure that contact resistances remain below $3 \mu \Omega$ for "half-moon" contacts, below $6 \mu \Omega$ for bus bar to heat sink contacts, and below $50 \mu \Omega$ for diode to heat sink contacts. Additionally the maximum allowed change of contact resistance between measurements was $1 \mu \Omega$ for total contact resistance in each lead and $0.5 \mu \Omega$ in case of "half-moon" contacts. In order to obtain contact resistance measurement precision better than $0.5 \mu \Omega$, the measurement system had to apply a DC current up to $5 \mathrm{~A}$. The resistance measurements included not only a contact which is being tested, but also resistance of part of the copper bus bar. Compensation of the copper bus bar resistance was necessary and had to be performed taking into account the temperature of the copper parts. The total number of performed diode lead contact resistance tests was 5887. Each test included measurements of resistances of all six contacts

\section{Identified Non-Conformities}

The results of failed electrical tests of all types of measurement campaigns were examined, and when needed additional measurements were performed. For instance in case of insulation failure detected during high voltage test described in [10] it was necessary to perform measurements of the breakdown signal propagation time in bus bars of affected circuits, during repeated high voltage insulation test, in order to localize the fault. Small number of tests may be failed due to external conditions, such as electromagnetic, thermal, or mechanical disturbance during high

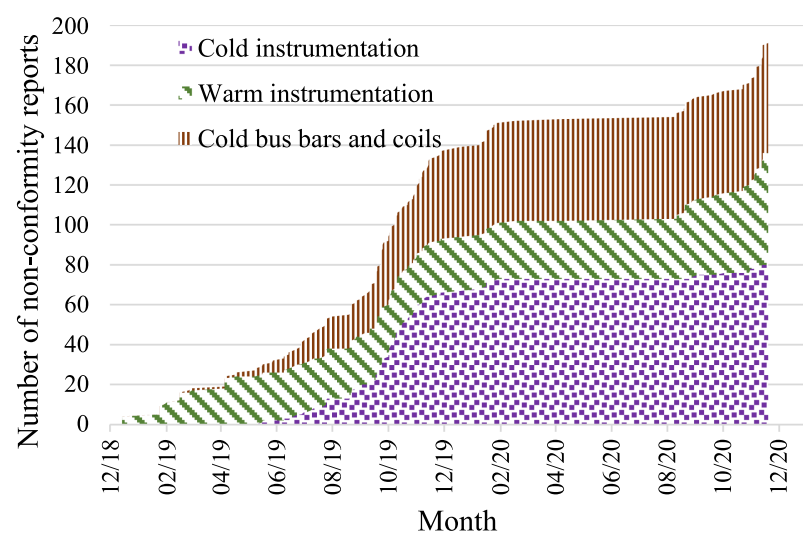

Fig. 3. Number of ELQA related non-conformity reports during LS2.

TABLE I

SUMmARY OF NON-CONFORMitIES REVEALED By NOVEMBER 2020

\begin{tabular}{cccc}
\hline \hline Fault type/Location & $\begin{array}{c}\text { Cold bus } \\
\text { bars, coils or } \\
\text { diodes }\end{array}$ & $\begin{array}{c}\text { Cold-side in- } \\
\text { strumentation }\end{array}$ & $\begin{array}{c}\text { Warm-side in- } \\
\text { strumentation }\end{array}$ \\
\hline Open circuit & 0 & 68 & 8 \\
Routing error & 1 & 0 & 1 \\
Abnormal resistance & 44 & 12 & 26 \\
Insulation failure & 10 & 0 & 8 \\
Mechanical damage & 1 & 67 & 16 \\
\hline
\end{tabular}

The fault types are not exclusive, e.g., open circuit can be caused by mechanical damage.

precision resistance measurement, resulting in abnormal readout, which is then detected by automatic validation procedure. In such case, test was repeated without creating a non-conformity report.

When a fault was revealed, a non-conformity report was created and followed-up. Eventually the affected component had to be repaired, replaced, bypassed, marked to be used as is, or locked out. Whenever a repair, replacement, or bypassing has been performed, the tests were repeated.

Electrical faults revealed by the ELQA measurements were located both in the main current carrying components of the circuits such as superconducting coils, bus bars and cold protection diodes, and in the warm and cold parts of the instrumentation. A total of 191 non-conformity reports were filled during LS2 by $29^{\text {th }}$ November 2020. The evolution of this number in time is presented in Fig. 3 and exact number of non-conformity reports, broken down into fault types and locations is shown in Table I.

An example of a non-conformity where open quench heater circuit was detected due to missing solder joint on the instrumentation feedthrough printed circuit board in one of the magnets installed during LS2 is presented in [11].

\section{CONCLUSION}

Currently the ELQA team is on the right path to finalize the qualification of the LHC superconducting circuits according to the LS2 planning. Thanks to efforts of CERN and IFJ PAN, it was possible to overcome the difficulties related to human resource management during the outbreak of the COVID-19 pandemic. ELQA tests allowed detection and treatment of non-conformities 
which otherwise could, in many cases, severely compromise performance of the LHC.

\section{ACKNOWLEDGMENT}

In this contribution we describe the collaborative effort between CERN and the Henryk Niewodniczanski Institute of Nuclear Physics Polish Academy of Sciences in Krakow during the three year-long preparation process and experiences related to the execution of the ELQA activities during LS2 of the LHC.

\section{REFERENCES}

[1] A. Kotarba et al., "Electrical quality assurance of the superconducting circuits during LHC machine assembly," in Proc. EPAC, Genoa, Italy, 2008, pp. 2440-2442.

[2] D. Bozzini et al., "Automatic system for the DC high voltage qualification of the superconducting electrical circuits of the LHC machine," in Proc. EPAC, Genoa, Italy, 2008, pp. 2416-2418.

[3] A. Kotarba et al., "Automatic measurement system for electrical verification of the LHC superconducting circuits," in Proc. IPAC, San Sebastian, Spain, 2011, pp. 1756-1758.

[4] M. Bednarek and J. Ludwin, "Software tools for the electrical quality assurance in the LHC," in Proc. ICALEPCS, Grenoble, France, 2011, pp. 993-995.
[5] R. Kulaga, Hardware and software upgrade of the high voltage crate for the ELQA, TE-MPE-TM meeting Feb. 21, 2013, Accessed: Oct. 1, 2020. [Online]. Available: https://indico.cern.ch/event/238924/

[6] J. Ludwin and P. Jurkiewicz, "Upgrade of the automatic measurement system for the electrical verification of the LHC superconducting circuits," IEEE Trans. Appl. Supercond., vol. 26, no. 3, Apr. 2016, Art. no. 0600803.

[7] M. Bednarski, P. Jurkiewicz, J. Ludwin, D. Wojas, M. Bednarek, and G. D'Angelo, "Upgrade of the arc interconnection verification system for the Large Hadron Collider," in Proc. MIXDES - 26th Int. Conf. Mixed Des. Integr. Circuits Syst., Rzeszow, Poland, 2019, pp. 106-110, doi: 10.23919/MIXDES.2019.8787158.

[8] J. Ph. Tock et al., "The second LHC long shutdown (LS2) for the superconducting magnets," in Proc. IPAC, Vancouver, BC, Canada, 2018, pp. 240-243, doi: 10.18429/JACoW-IPAC2018-MOPMF056.

[9] D. Wojas, Development of new diode lead measurement (DLM) system for QA of DISMAC project, TE-MPE-TM meeting Apr. 18, 2019, Accessed: Oct. 254 1, 2020. [Online]. Available: https://indico.cern.ch/event/ 813875/

[10] M. Bednarek, G. D'Angelo, J. Ludwin, and D. Wojas, Nonconformity Report LHC-QN-ELQA-TP4B-HVQ-RCBXV1.L2-001, Nov. 13, 2020, Accessed: Feb. 9, 2021. [Online]. Available: https://edms.cern.ch/document/ 2439966/1

[11] G. D'Angelo and D. Wojas, Nonconformity Report LHC-QN-ELQAMIC-W-QHR-MBBL2303-A20L3-001, Nov. 6, 2020, Accessed: Feb. 9, 2021. [Online]. Available: https://edms.cern.ch/document/2435976/1 